\title{
PRESENCIA DE MERCURIO EN EL AGUA Y SEDIMENTO DE FONDO EN EL RÍO NANAY, PERÚ
}

\author{
PRESENCE OF MERCURY IN THE BOTTOM SEDIMENTS AND WATERS \\ IN THE NANAY RIVER, PERU
}

\author{
José Maco García ${ }^{1}$ y Elvis Sandoval Zamora²
}

\begin{abstract}
RESUMEN
Evaluaciones de mercurio en el agua y sedimentos de fondo del río Nanay, afluente izquierdo del río Amazonas, ha determinado un claro incremento de las concentraciones promedio que oscilan entre $<20 \mathrm{ng} / \mathrm{l}$ en el año 2000 a 120 ng/l en el 2001. Se identifican lugares con concentraciones superiores a los límites máximos permisibles para aguas de zonas de pesca de mariscos bivalvos (Tipo V) y aguas de zonas de preservación de fauna acuática y pesca recreativa o comercial (Tipo VI) regulados en el Perú. Los sedimentos de fondo presentan concentraciones entre 0.005 a 0.0815 ìg/g, valores considerados por debajo de los límites máximos permisibles establecidos por la EPA(Environmental Protection Agency). En general las fuentes de mercurio del agua provienen parcialmente de los sedimentos del fondo del río debido a su remoción durante la extracción del oro aluvial.
\end{abstract}

Palabras clave: contaminación, mercurio, río Nanay, agua, sedimentos, Amazonía, Perú.

\begin{abstract}
Measurements of mercury in the water and sediments of Nanay river bottom, left affluent of the Amazon river, has determined a clear average increasing of concentrations that range between $<20 \mathrm{ng} / \mathrm{l}$ in the year 2000 to $120 \mathrm{ng} /$ $l$ in the year 2001. There were identified places with concentrations excceding the maximum permissable limits for waters of zones for bivalve seafood fishing (Type V) and waters zones for preservation of aquatical fauna and recreative or commercial fishing (Type VI) regulated by Peruvian law. The sediments of river bottom have concentrations between 0.005 to $0.0815 \mu \mathrm{g} / \mathrm{g}$, values considered below the maximum permissable limits established by the EPA (Environmental Protection Agency). In general, the mercury sources of the water partially become from the sediments of the river bottom due to their removal during the extraction of the alluvial gold.
\end{abstract}

Key words: pollution, mercury, Nanay river, water, sediments, Amazonia, Peru.

\section{INTRODUCCIÓN}

El mercurio, metal muy tóxico para el ambiente y la salud de las poblaciones humanas, es objeto de estudio en varios lugares de la cuenca del río Amazonas (Veiga 1997a; 1997b; Lodenius y Malm 1998; Maurice-Bourgoin et al. 1999; Roulet et al. 1999; 2000a; 2000b, 2001a; 2001b; Carmouze et al. 2001).

En la Amazonía peruana se han realizado muy pocos estudios sobre los niveles de contaminación del mercurio. Algunos estudios se realizaron en la cuenca del río Madre de Dios, donde se sabe que existe una importante actividad minera aurífera en las zonas aluviales que causa serios daños al ambiente y a la salud de la población. Hasta 1994 participaron 3,000 personas en la actividad aurífera en Madre de Dios (GRADE 1994), mientras que en 1999 ese número se elevó a 10,000 personas que trabajan directamente en la minería, en las pequeñas cuencas de Huaypetuhe y Caychive en la cuenca del Madre de Dios (Lundberg 1999). Según IMA (1995) se empleó más de $90 \mathrm{t}$ de mercurio en ocho años de actividad. Hasta 1996, el contenido de mercurio en los cuerpos de agua de la cuenca del Madre de Dios fue de 0.6 a $1.5 \mathrm{ng} / \mathrm{L}$, mientras que los niveles de mercurio en los sedimentos de la cuenca, son del orden de 0.156 a 0.358 ?g/g (Deza 1996).

1 Investigador del Instituto de Investigaciones de la Amazonía Peruana Av. Abelardo Quiñones km 2.5. Apartado 784. Iquitos

- Perú. Correo electrónico: jmaco@iiap.org.pe

2 Funcionario de la Dirección Ejecutiva de Salud Ambiental de Loreto. 
La cuenca del río Nanay es una de las principales fuentes de abastecimiento de alimentos y de aprovisionamiento de agua, la misma que luego de ser potabilizada es distribuida a la ciudad de Iquitos, que alberga una población cercana al de medio millón de habitantes. Esta cuenca es soporte de las actividades socioeconómicas de aproximadamente 25,200 habitantes rurales que se ubican en las orillas del mismo río y sus principales afluentes. En este marco, toda perturbación ambiental en ese importante espacio geográfico, de 1’750,737 ha (IIAP 2002), tiene serias implicaciones regionales, por lo que el desarrollo de las actividades humanas en esta cuenca deben realizarse de forma sostenible teniendo los cuidados necesarios para minimizar la degradación ambiental. En 1994 se realizaron determinaciones de mercurio en el río Nanay, dando como resultado la ausencia de este metal en el agua (Gómez 1994). En esa oportunidad el mercurio no fue detectado, posiblemente debido a las limitaciones referentes a la precisión del método empleado.

A partir de 1999, se reporta el desarrollo de la actividad minera en la cuenca del río Nanay donde se identifican una serie de impactos negativos para el medio ambiente y la salud humana durante el proceso de extracción de minerales (Reyes 2000). Uno de los impactos más severos que la actividad aurífera ocasiona está relacionado con el uso de mercurio durante el proceso de la extracción de oro; el mercurio es un metal sumamente tóxico (Español 2001; Goldman et al. 2001; Clarkson 2002; Kales \& Goldman 2002 y otros). Conocedores de esta problemática, se conformó en la región Loreto una Comisión Técnica Multiinstitucional con la finalidad de evaluar los impactos ocasionados por la extracción de oro en la cuenca del río Nanay. El presente documento incluye parte de los análisis de los estudios realizados por dicha Comisión durante los años 2000 y 2001. Aquí se exponen los resultados de los análisis de mercurio en agua y sedimento de la cuenca del río Nanay.

El río Nanay es el eje hídrico principal de la cuenca del Nanay que está localizada en el llano amazónico, en la parte noreste del territorio del Perú. Pertenece a la provincia de Maynas, región Loreto, y geográficamente se encuentra ubicada entre los meridianos $73^{\circ} 13^{\prime}$ y $75^{\circ} 03^{\prime}$ longitud oeste y los paralelos $2^{\circ} 33^{\prime}$ y $4^{\circ} 02^{\prime}$ latitud sur (figura 1 ).

El río Nanay nace en el llano amazónico y desemboca en la margen izquierda del río Amazonas a la altura de la ciudad de Iquitos. Es un río pequeño cuyo caudal oscila entre 70 a 200 m³/s (AUDITEC SAC-DINAMICA 2000). Presenta características limnológicas que corresponden a aguas negras como las descritas por Sioli (1984), las mismas que se resumen en tener moderada transparencia, alrededor de un metro de profundidad de la columna de agua, oxígeno disuelto de 2.5 a 4.7 mg/L, un pH ligeramente ácido de 6.3 a 6.6 (Gómez 1994) y coloración negruzca; su caudal es de alrededor de 240 m³/s (IIAP 1999a; 1999b; 2002).

En el ámbito de su cuenca se realizan una serie de actividades económicas, destacando las siguientes: la extracción de madera y de productos diferentes a la madera, agricultura de subsistencia, caza de subsistencia, pesca de subsistencia y comercial, así como la extracción oro aluvial y material de acarreo. La extracción de estos minerales comenzó en 1999 (Reyes 2000) empleándose para el efecto «dragas de succión», que remueven el fondo del río hasta una profundidad de un metro. De acuerdo a los estudios de impacto ambiental, las dragas tienen una capacidad de succión de $60 \mathrm{~m}^{3} /$ hora de sedimentos y trabajan un promedio de 15 horas por día (AUDITEC SACDINAMICA 2000). Sin embargo, los pobladores del río Nanay mencionan que las dragas trabajaban las 24 horas del día. En el río Nanay, la ley del oro es de alrededor de $0.3 \mathrm{~g} / \mathrm{m}^{3}$. y durante el proceso de amalgamado se usa mercurio metálico, en cantidades que oscilan entre dos a tres veces la cantidad de oro encontrado. 


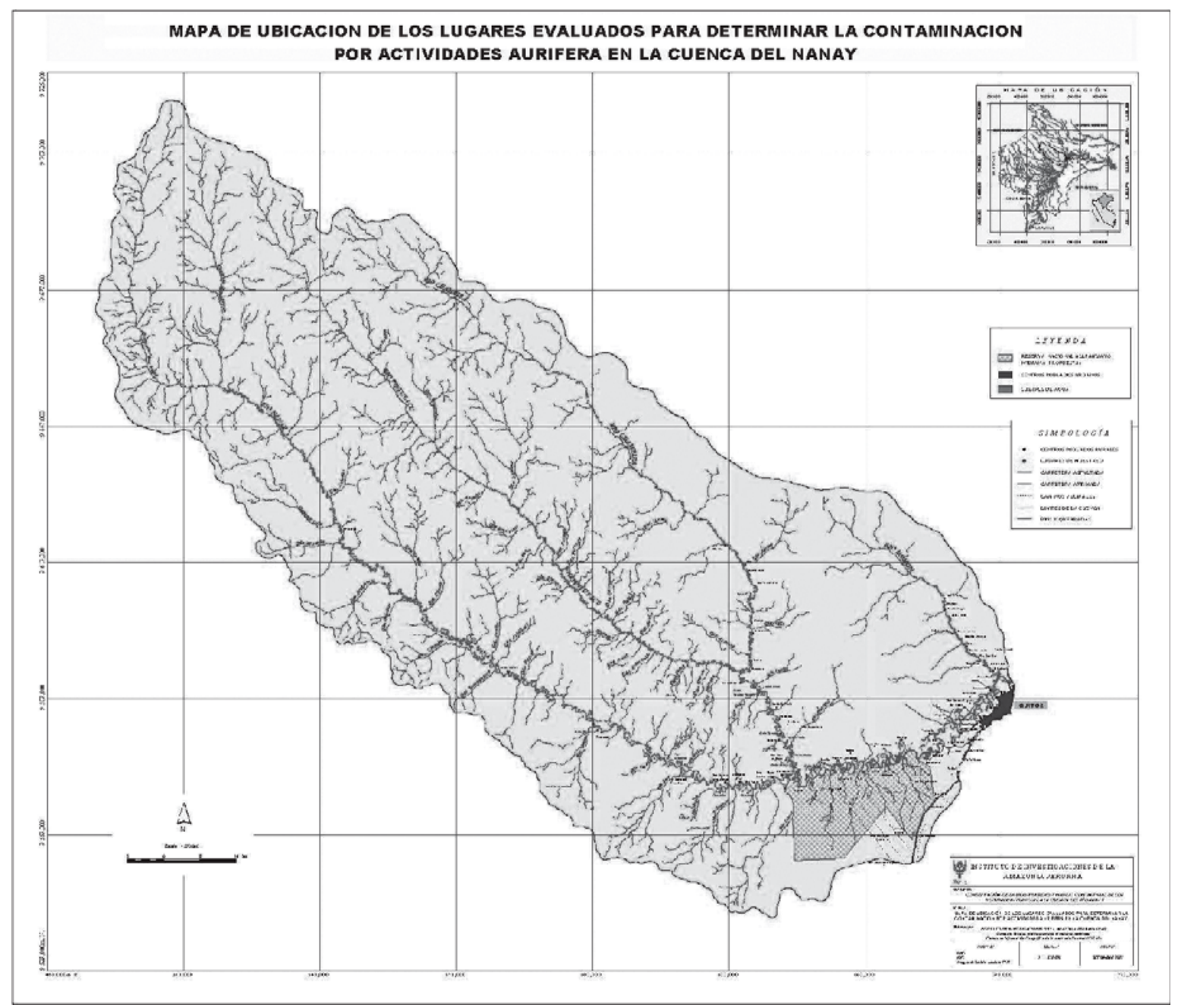

Figura 1.

\section{MATERIAL Y MÉTODO}

La toma de muestras del río Nanay se realizó durante mayo de 2000, y mayo y octubre de 2001. En el 2002, se establecieron cuatro estaciones de muestreo donde se colectó una muestra de agua del centro del río, por estación. Durante el año 2001 se establecieron 15 estaciones de muestreo y se tomó una muestra de agua del centro del río por estación. Las muestras de agua fueron colectadas en botellas de teflón de un litro de capacidad y fueron adecuadamente preservadas con $1 \mathrm{ml}$ de ácido nítrico concentrado y refrigeradas a $4^{\circ} \mathrm{C}$ hasta su envío al laboratorio.

Solamente en octubre de 2001, paralelamente a la toma de muestras de agua, se colectaron muestras de sedimentos del lecho del río en las 15 estaciones de muestreo. Se empleó una draga tipo Eckman y las muestras fueron colocadas en bolsas de polietileno, etiquetadas y refrigeradas a $4^{\circ} \mathrm{C}$ hasta su envío al laboratorio.

Posteriormente, las muestras de agua y sedimentos colectados fueron remitidos a la ciudad de Lima para ser analizados en los laboratorios de la Dirección General de Salud Ambiental-DIGESA del Ministerio de Salud del Perú. El análisis de mercurio se realizó a través del método Oficial Method AOAC 986.15 Multielement Methods: arrastre por vapor frío sin flama - absorción atómica. 


\section{RESULTADOS}

\section{Mercurio en agua}

La concentración de mercurio total medido en las aguas superficiales del río Nanay se incrementa a través del tiempo. En los muestreos realizados durante el año 2000, las concentraciones de mercurio total fueron menores de 20 ng/L. Estos resultados son inferiores a las concentraciones de los límites máximos permisibles establecidos por el Perú para los diferentes tipos de agua de acuerdo a sus usos (El Peruano 1983). Esos límites oscilan entre 100 ng/L y 2,000 ng/L de mercurio. Sin embargo, en el 2001 las concentraciones de mercurio en la cuenca del río Nanay se incrementaron (figuras 2 y 3), llegando en algunos casos a sobrepasar los valores de los límites máximos permisibles para los tipos de agua V (aguas de zonas de pesca de mariscos bivalvos) y VI (aguas de zonas de preservación de fauna acuática y pesca recreativa o comercial).

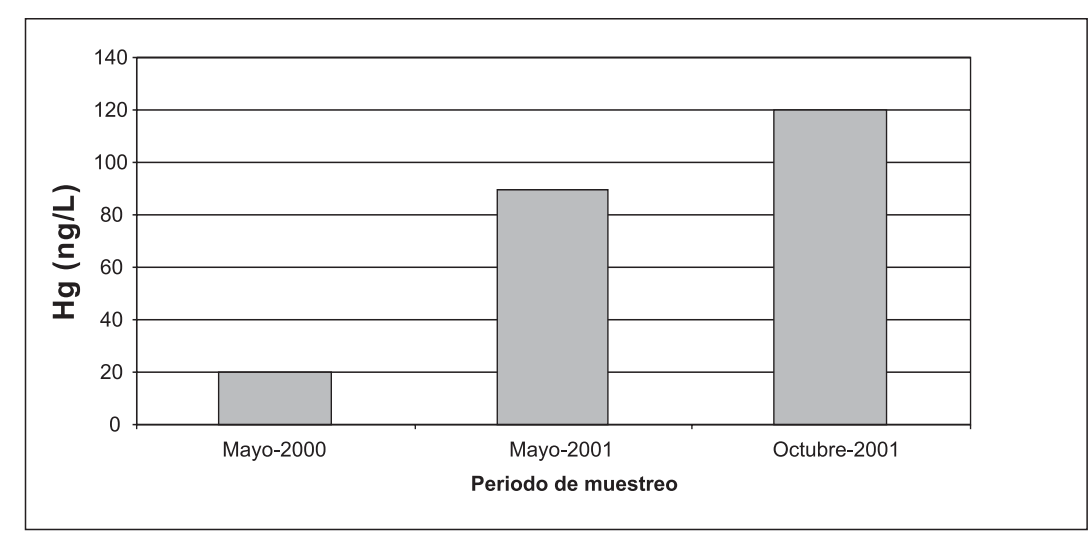

\section{Nótese el incremento de mercurio del año 2000 al 2001 en el río Nanay.}

Figura 2. Contenido promedio de mercurio en agua (mg/L) del río Nanay. Fuente: DIGESA

En el mes de mayo del 2001, que corresponde al periodo de creciente del río, la concentración de mercurio en el río Nanay varió entre <20 a 920 ng/L, siendo el promedio de 90 ng/L (n=15). Durante el mes de octubre de 2001, correspondiente al periodo de vaciante del río, la concentración de mercurio en el río Nanay fue de $<20$ a 230 ng/ $\mathrm{L}$, con promedio de $120 \mathrm{ng} / \mathrm{L}(\mathrm{n}=15)$. Las mayores concentraciones de mercurio en el río Nanay se encuentran cerca de la ciudad de Iquitos (figura 1). 


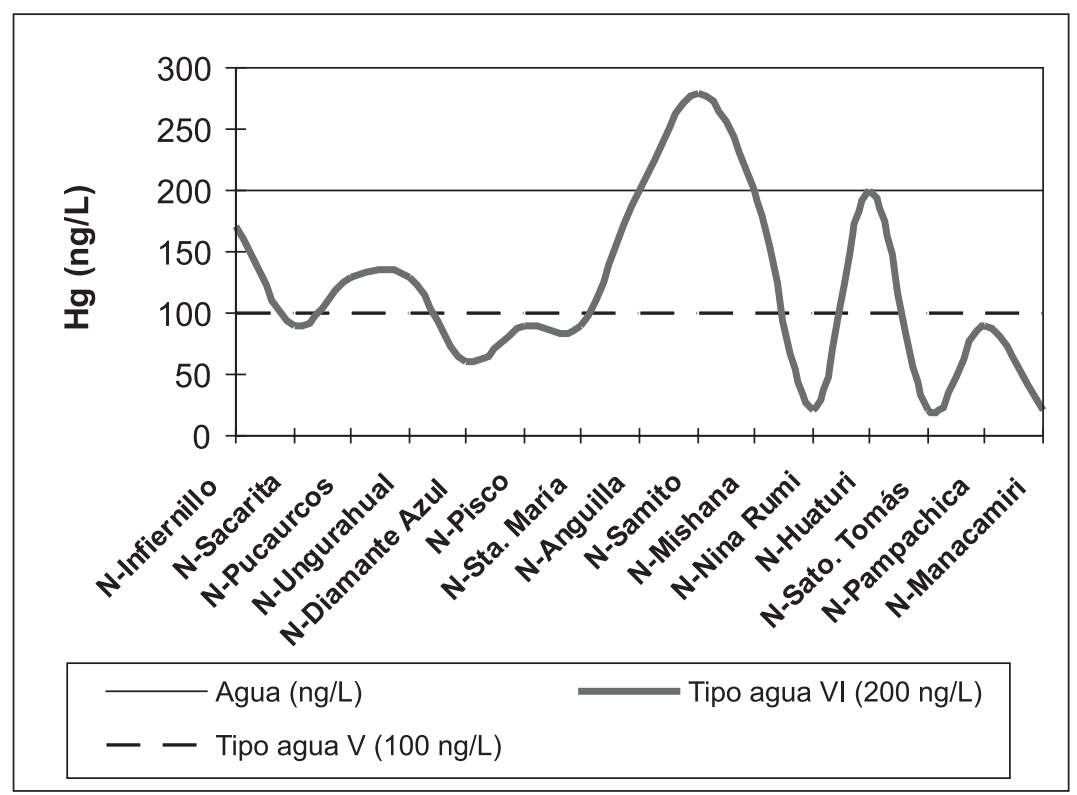

Nótese que algunas muestras de agua sobrepasan los límites máximos permisibles para los tipos de agua Vy VI de la Ley general de Aguas del Perú.

Figura 3. Contenido de mercurio en agua (mg/L) del río Nanay en el mes de octubre del 2001. Fuente: DIGESA

\section{Mercurio en sedimentos del fondo del río}

En las muestras de sedimentos del fondo del río Nanay colectadas en octubre de 2001, los niveles de mercurio oscilaron entre 0.005 a 0.0815 ìg/g, con un promedio de 0.0249 ìg/g; observándose un incremento de la concentración de mercurio río abajo como se muestra en la figura 4. Estos valores de mercurio no sobrepasan los límites máximos permisibles de 0.2 ìg/g, establecidos por la EPA. Las leyes peruanas no contemplan límites máximos permisibles de mercurio para este componente. 


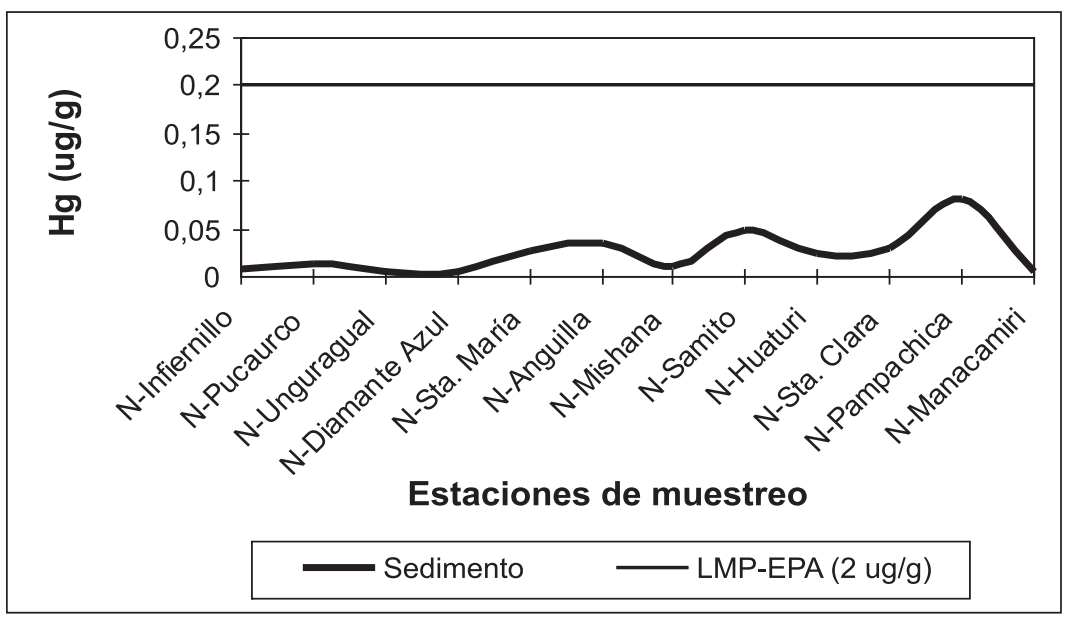

Las muestras de sedimentos no sobrepasan el límite máximo permisible (LMP) establecidos por Ia EPA.

Figura 4. Contenido de mercurio en sedimento de fondo del río Nanay (ìg/g) en el mes de octubre de 2001. Fuente: DIGESA

\section{Relación sedimento-agua}

Con la finalidad de tratar de explicar la presencia de mercurio en el agua se ha establecido una relación entre los niveles de mercurio encontrados en los sedimentos y en el agua del río Nanay (figura 5). Se observa que la concentración de mercurio en los sedimentos explican parcialmente, la presencia de las concentraciones de mercurio encontradas en el agua $(\mathrm{r}=0.158)$. En este contexto, el mercurio encontrado en el agua, además de provenir de los sedimento del río, podría provenir de otras fuentes.

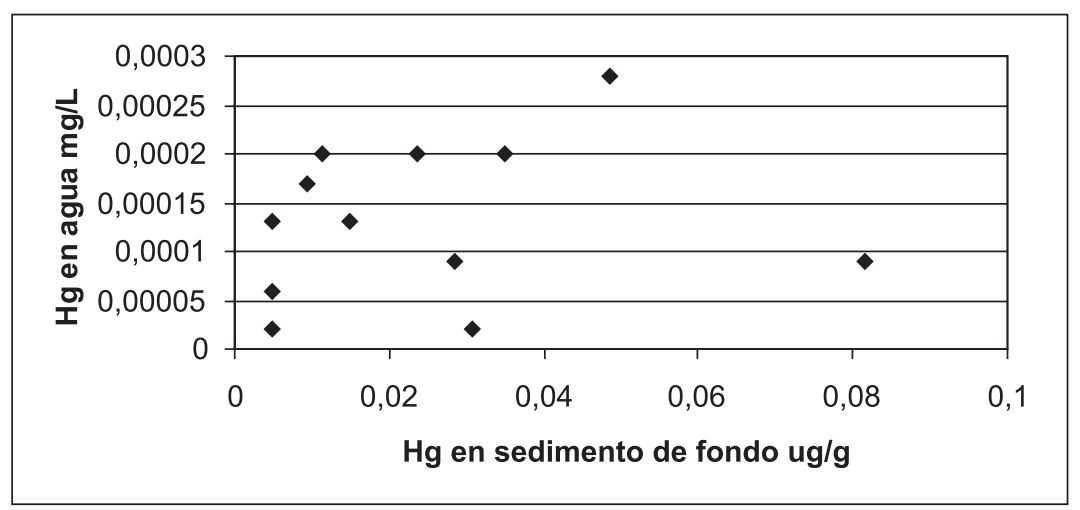

Nótese que algunas muestras de agua sobrepasan los límites máximos permisibles para los tipos de agua Vy VI de la Ley General de Aguas.

Figura 5. Relación de los contenidos de mercurio en agua (mg/L) y en los sedimentos (ìg/g) en el río Nanay durante el mes de octubre de 2001.

Fuente: DIGESA 


\section{DISCUSIÓN}

Los niveles de mercurio encontrados en el río Nanay son superiores a los valores de mercurio reportados para otros ríos de la Amazonía, como por ejemplo los ríos de agua blanca como el río Madre de Dios, Perú (Deza 1996) y el río Beni, Bolivia (Maurice-Bourgoin et al. 1999), en los que se realiza una importante actividad minera. Concentraciones de mercurio menores a las del río Nanay han sido encontrados también en el río Amazonas, en territorio brasileño (Roulet et al. 2001b). En estos ríos de agua blanca las mayores concentraciones de mercurio se encuentran en las partículas en suspensión (Roulet et al. 2001b). En el río Madre de Dios, estas partículas en suspensión compuestas mayormente de arena, arcilla y limo proporcionan al agua una coloración marrón y escasa transparencia de la columna de agua de alrededor de 16 a 24 cm (IIAP-CTAR Madre de Dios 2000). En cambio, el río Nanay presenta características limnológicas de agua negra con buena transparencia debido a que las partículas en suspensión son escasas (IIAP 1999a; 1999b; 2002) de ahí que la mayor concentración de mercurio se encuentre en el agua.

Las altas concentraciones de mercurio en el río Nanay también pueden estar relacionadas con la magnitud de su caudal. El Nanay presenta un caudal muy reducido (alrededor de 240 m³/s; IIAP 1999a; 1999b; 2002) con relación a los ríos de agua blanca anteriormente descritos, como por ejemplo, el río Madre de Dios, que en el lado peruano presenta un caudal de 9,281 m³/s (IIAP-CTAR Madre de Dios 2000).

De acuerdo a la legislación peruana, en el río Nanay existen algunos lugares donde se presentan niveles de mercurio superiores a los límites máximos permisibles para dos tipos de uso de aguas (El Peruano 1983). Los niveles de mercurio en el río Nanay no permitirían realizar el aprovechamiento de algunos recursos hidrobiológicos, tal es el caso de los moluscos bivalvos para los cuales se ha establecido que las zonas de pesca de estos moluscos no debe de sobrepasar los $100 \mathrm{ng} / \mathrm{L}$ de mercurio (agua de tipo de uso V). En este contexto, la mayoría de los lugares muestreados presentan niveles de mercurio que sobrepasan el nivel antes señalado (figura 3). En el río Nanay existen algunos lugares de muestreo donde los niveles de mercurio sobrepasan los $200 \mathrm{ng} / \mathrm{L}$ de mercurio, nivel que es establecido como límite máximo permisible para las zonas de preservación de fauna acuática y pesca recreativa o comercial (agua de tipo de uso VI, figura 3).

Para otros usos del agua del río Nanay, como el abastecimiento de agua potable para la ciudad de Iquitos, los niveles de mercurio son muy bajos y, aparentemente, no son perjudiciales para la población iquiteña. Sin embargo, más de 25,000 habitantes de las poblaciones ribereñas consumen diariamente el agua en forma directa, sin ningún tratamiento de potabilización. En este marco, se calcula una ingesta diaria promedio de mercurio de 0.36 ìg/L y mensual de 10.8 ìg/L, considerando, por un lado, la ingesta diaria de tres litros de agua por habitante como promedio, ya para calmar la sed con agua, refresco u otro o de agua usada para la preparación de los alimentos.

Con relación a los sedimentos del fondo del río Nanay, las concentraciones de mercurio son inferiores a las encontradas para otros ambientes acuáticos de la Amazonía, como por ejemplo para el río Madre de Dios, Perú (Mora 1995, citado en Deza 1996) y para el río Madeira, Brasil (Malm et al. 1995 y Martinelli et al. 1988, citados en Deza 1996). Este hecho corrobora la tesis de que en la Amazonía se ha identificado como una de las fuentes de mercurio a los sedimentos acarreados de las laderas de los Andes (Maurice-Bourgoin et al. 1999; Roulet et al. 2001a). Este es el caso de los ríos de agua blanca mencionados anteriormente, pero no lo es para el río Nanay, que tiene origen en el llano amazónico y los sedimentos escasos que acarrea son provenientes del bosque de tierra firme, de las zonas hidromórficas y de las áreas que inunda periódicamente durante el periodo de creciente.

Considerando las concentraciones de mercurio en los sedimentos y las cantidades del mismo que son removidas por dragas (600 m³/día, de acuerdo a los estudios de impacto ambiental AUDITEC SAC-DINAMICA 2000), se realizaron cálculos sobre liberación de mercurio provenientes de los sedimentos del fondo del río. Si consideramos la concentración promedio de 0.0249 ìg/g de mercurio en el sedimento, una draga estaría removiendo 1,328 mg de mercurio en los ocho meses de trabajo al año en los que el nivel de las aguas es adecuado para la realización de esa actividad. 


\section{CONCLUSIONES}

Los niveles promedio del contenido de mercurio en el río Nanay se incrementan de <20 ng/L en el 2000 a 120 ng/ L en el 2001. En este río se registran concentraciones de mercurio superiores a los límites máximos permisibles para los tipos de agua V (aguas de zonas de pesca de mariscos bivalvos) y VI (aguas de zonas de preservación de fauna acuática y pesca recreativa o comercial) establecidos en las leyes peruanas (El Peruano 1983). En los sedimentos de fondo se presentan concentraciones de mercurio que oscilan entre 0.005 a $0.0815 \mathrm{~g} g / \mathrm{g}$, valores que se mantienen por debajo de los límites máximos permisibles establecidos por la EPA. No obstante, se infiere una importe fuente de mercurio proporcionado por la actividad minera que se desarrolla en la cuenca, la misma que realiza una fuerte remoción de sedimentos del fondo del río, afectando a diferentes nichos ecológicos, en perjuicio directo de las especies bentónicas.

En este contexto, se puede predecir que la continuación e incremento de las actividades de extracción minera, especialmente la aurífera, en la cuenca del río Nanay, producirá mayores impactos en perjuicio de la conservación de la cuenca y el uso sostenible de sus recursos naturales.

\section{RECONOCIMIENTO}

A las instituciones integrantes de la Comisión Técnica Multisectorial para la evaluación de las actividades auríferas en el río Nanay por su participación en los periodos de muestreo; al IIAP y a la Comisión Ambiental de Loreto por el apoyo decidido para la realización del estudio y a la Dirección General de Salud Ambiental-DIGESA, por la realización de los análisis de mercurio en agua y sedimentos del fondo del río.

\section{BIBLIOGRAFÍA}

AUDITEC SAC-DINAMICA. 2000. Estudio de Impacto Ambiental: Dragado en el río Nanay. Dinámica Minera y Agroindustrial S.A.-DIMIASA. Ministerio de Energía y Minas Dirección General de Asuntos Ambientales. Loreto-Perú.

CARMOUZE, J.P., M. LUCOTTE AND A. BOUDOU. 2001. Mercury en the Amazon: Importance of human and environment, health hazards. IRD Editions. París. 40 pp.

CLARKSON, T.W. 2002. The three modern faces of mercury. Environmental Health Perspectives, 110(1): 11-22.

DEZA, N.E. 1996. Mercury acumulation in fish from Madre de Dios, a goldminig area in the Amazon basin, Perú. Thesis of Master of Science. Oregon. 39 p.

EL PERUANO. 1983. Decreto Supremo n. 007-83-SA. 17 de marzo de 1983: 14698-14700.

ESPAÑOL, C. S. 2001. Toxicología del mercurio. Actuaciones preventivas en sanidad laboral y ambiental. CD: Jornada internacional sobre impacto ambiental del mercurio utilizado por la minería aurífera artesanal en Iberoamérica. Setiembre de 2001. Lima-Perú. 69 p.

FAO 1983. Compilation of legal limits for Hazardous Sunstances in Fish and Fishery Products. FAO. Fisheries Circular n. ${ }^{\circ}$ 764. Roma.

GÓMEZ, R. 1994. Contaminación ambiental en la Amazonía peruana. Reporte 1994. Informe técnico de avance. Instituto de Investigaciones de la Amazonía Peruana. Iquitos-Perú. 52 p. + anexos. 
GOLDMAN, L. R., M.W. SHANNONAND THE COMMITTEE ON ENVIRONMENTAL HEALTH. 2001. Technical Report: Mercury in the Environment: Implications for Pediatricians. American Academy of Pediatrics. PEDIATRICS, 108(1): 197-205.

GRADE, 1994. Estudio de la minería informal y el medio ambiente (Sub Proyecto MIMA) Zona: Madre de Dios. Informe final. Programa de Asistencia Técnica al Sector Energía y Minas. 135 pp.

IIAP (Instituto de Investigaciones de la Amazonía Peruana). 1999a. Caracterización Física de la cuenca del río Nanay. Proyecto Integral y Sostenible del río Nanay de la Amazonía Peruana. Programa de Ordenamiento Ambiental. Iquitos. 14 pp.

IIAP (Instituto de Investigaciones de la Amazonía Peruana). 1999b. Informe final proyecto «Evaluación de la fauna silvestre en el área de influencia de la carretera Iquitos - Nauta» y propuesta de un plan de manejo de la fauna silvestre. Iquitos. 78 pp.

IIAP (Instituto de Investigaciones de la Amazonía Peruana). 2000a. Evaluación y conservación de la diversidad biológica para el ecoturismo. Informe Final del Proyecto. Iquitos.92 p.

IIAP (Instituto de Investigaciones de la Amazonía Peruana). 2000b. Caracterización de ecosistemas para protección y uso de la cuenca del río Nanay. Conservación y uso de ecosistemas. Informe Anual. 62 p.

IIAP (Instituto de Investigaciones de la Amazonía Peruana). 2002. Propuesta de Zonificación Ecológica Económica de la cuenca del río Nanay. Programa de Ordenamiento Ambiental-Proyecto Conservación de la Biodiversidad y Manejo Comunitario de los Recursos Naturales de la Cuenca del Río Nanay. Convenio IIAP-Banco Mundial. Tipog. 111 p.

IIAP (Instituto de Investigaciones de la Amazonía Peruana)-CTAR Madre de Dios (Consejo Transitorio de Administración Regional de Madre de Dios). 2000. Medio físico, zonificación ecológica económica de la región Madre de Dios. Volumen II, Puerto Maldonado, 151 p.

IMA (Instituto de Manejo de Agua y Medio Ambiente). 1995. Efectos de la contaminación por mercurio en la explotación de oro en Madre de Dios. Cusco. 45 p.

KALES, S.N. AND R.H. GOLDMAN. 2002. Mercury Exposure: Current concepts, controversies and a Clinic's experience. Jornal of Ocupational and Environmental Medicine, 44(2).

LACERDA, L.D. 1995. Amazon mercury emissions. Nature, 374: 20-21.

LODENIUS, M. AND O. MALM. 1998. Mercury in the Amazon. Rev. Environ. Contam. Toxicol, 157: 25-52.

LUNDBERG, B. 1999. Actividad aurífera y medio ambiente: con comentarios sobre el trabajo de zonificación. Programa de zonificación ecológica económica para el desarrollo sostenible de la cuenca del río Madre de Dios. Tipog. 16 p.

MAURICE-BOURGOIN, L., I. QUIROGA, J.L. GUYOTAND O. MALM. 1999. Mercury pollution in the upper Beni River, Amazonian Basin: Bolivia. Ambio 28 (4):302-306.

REYES, F.C. 2000. Explotación de oro aluvial en el río Nanay. Informe s/n-2000-CTAR-L-ST/CRF. Tipog. 20 pp.

ROULET, M., J. R. D. GUIMARÃES AND M. LUCOTTE. 2001. Methylmercury production and accumulation in sediments and soils of Amazonian floodplain - Effect seasonal inundation. Water, Air, and Soil Pollution, 128: 41-60. 
ROULET, M., M. LUCOTTE, R. CANUEL, N. FARELLA, M. COURCELLES, J.R.D. GUIMARÃES, D. MERGLER AND M. AMORIM. 2000a. Increase in mercury contamination recorded in lacustrine sediments following deforestation in the central Amazon. Chemical Geology, 165: 243-266.

ROULET, M., M. LUCOTTE, R. CANUEL, N. FARELLA, G. Y. G. DE FREITOS, P. J. R. PACHECO, J.R.D. GUIMARÃES, D. MERGLER AND M. AMORIM, 2001b. Spatio-temporal geochemistry of mercury in waters of the Tapajós and Amazon rivers, Brazil. Limnol. Oceanogr., 46(5): 1141-1157.

ROULET, M., M. LUCOTTE, N. FARELLA, G. SERIQUE, H. COELHO, C.J. SOUSA PASSOS, E. DE JESÚS DA SILVA, P. SCAVONE DE ANDRADE, D. MERGLER, J. R. D. GUIMARÃES AND M. AMORIM. 1999. Effects of recent human colonization on the presence of mercury in Amazonian ecosystems. Water, Air, and Soil Pollution, 112: 297-313.

ROULET, M.; M. LUCOTTE, J. R. D. GUIMARÃES AND I. RHEAULT. 2000b. Methylmercury in water, seston and epiphyton of an Amazonian river and its floodplain, Tapajós River, Brazil. The Science of the Total Environment 261: 43-59.

SIOLI, H. (ed.). 1984. The Amazon. Linmology and landscape ecology of a mighthy tropical river and its. Dr. Junk Publishers, Dordrecht. 763 p.

VEIGA, M.M. 1997a. Introducing new technologies for abatement of global mercury pollution in Latin America. Rio de Janeiro: UNIDO/UBC/CETEM/CNPq. 64 p.

VEIGA, M.M. 1997b. Mercury in artisanal gold mining in Latin Amarica: facts, fantasies and solutions. UNIDOExpert Group Meeting-Introducting new technologies for abatement of global mercury pollution deriving from artisanal gold mining. Vienna. 23 p. 